ARTICLE

\title{
Direct exfoliation and dispersion of two-dimensional materials in pure water via temperature control
}

Jinseon Kim ${ }^{1, \star}$, Sanghyuk Kwon ${ }^{2, \star}$, Dae-Hyun Cho ${ }^{3}$, Byunggil Kang ${ }^{4}$, Hyukjoon Kwon ${ }^{4}$, Youngchan Kim ${ }^{4}$, Sung O. Park ${ }^{5}$, Gwan Yeong Jung ${ }^{5}$, Eunhye Shin ${ }^{5}$, Wan-Gu Kim ${ }^{6}$, Hyungdong Lee ${ }^{2}$, Gyeong Hee Ryu ${ }^{7}$, Minseok Choi ${ }^{8}$, Tae Hyeong Kim ${ }^{8}$, Junghoon Oh${ }^{9}$, Sungjin Park ${ }^{9}$, Sang Kyu Kwak ${ }^{5}$, Suk Wang Yoon ${ }^{6}$, Doyoung Byun ${ }^{2}$, Zonghoon Lee ${ }^{7} \&$ Changgu Lee 2,4

The high-volume synthesis of two-dimensional (2D) materials in the form of platelets is desirable for various applications. While water is considered an ideal dispersion medium, due to its abundance and low cost, the hydrophobicity of platelet surfaces has prohibited its widespread use. Here we exfoliate 2D materials directly in pure water without using any chemicals or surfactants. In order to exfoliate and disperse the materials in water, we elevate the temperature of the sonication bath, and introduce energy via the dissipation of sonic waves. Storage stability greater than one month is achieved through the maintenance of high temperatures, and through atomic and molecular level simulations, we further discover that good solubility in water is maintained due to the presence of platelet surface charges as a result of edge functionalization or intrinsic polarity. Finally, we demonstrate inkjet printing on hard and flexible substrates as a potential application of water-dispersed 2D materials.

\footnotetext{
${ }^{1}$ Mechanical Test \& Analysis Section, Korea Electric Power Corporation Nuclear Fuel, Daedeok-daero 989 beon-gil, Youseong-gu, Daejeon 305-353, Korea. ${ }^{2}$ School of Mechanical Engineering, Sungkyunkwan University, 2066 Seobu-ro, Jangan-gu, Suwon 440-746, Korea. ${ }^{3}$ Department of Mechanical Engineering, The Ohio State University, 281 W. Lane Ave. Columbus, Ohio 43210, USA. ${ }^{4}$ SKKU Advanced Institute of Nanotechnology, Sungkyunkwan University, 2066 Seobu-ro, Jangan-gu, Suwon 440-746, Korea. ${ }^{5}$ School of Energy and Chemical Engineering, Ulsan National Institute of Science and Technology, Ulsan 689-798, Korea. ${ }^{6}$ Department of Physics, Sungkyunkwan University, 2066 Seobu-ro, Jangan-gu, Suwon 440-746,Korea. ${ }^{7}$ School of Materials Science and Engineering, Ulsan National Institute of Science and Technology, Ulsan 689-798, Korea. ${ }^{8}$ New Material Team, Future Device R\&D Department, LG Electronics Advanced Research Institute, 38 Baumoe-ro, Seocho-gu, Seoul 137-724, Korea. ${ }^{9}$ Department of Chemistry and Chemical Engineering, Inha University, Incheon 402-751, Korea.

*These authors contributed equally to this work. Correspondence and requests for materials should be addressed to C.L. (email: peterlee@skku.edu).
} 
$\mathrm{T}$ wo-dimensional (2D) sheets of graphene, hexagonal boron nitride (h-BN) and $\mathrm{MoS}_{2}$ have been studied vigorously in recent years because of their attractive mechanical, electrical, optical and chemical properties ${ }^{1-6}$. Although these materials all have atomic-level thicknesses, they have different physical properties, and so can complement each other in many ways. For example, because graphene is a conductor, h-BN an insulator, and $\mathrm{MoS}_{2}$ a semiconductor, they can be combined to fabricate field-effect transistors or electric circuits consisting purely of $2 \mathrm{D}$ materials ${ }^{7}$. In mechanical applications, they can be used as lubricating coatings or composites either with or without electric conductivity depending on their electrical properties. In numerous applications, such as batteries, surface coatings, composites, solar cells and catalysts, they need to be synthesized with platelet shapes in large quantities and most preferably at low cost. Dispersion in solvents, and particularly in cheap solvents, is the best approach to material preparation so as to satisfy these conditions. Since the surfaces of these materials are mostly hydrophobic ${ }^{8}$, it is difficult to exfoliate and dissolve them directly in water without the use of chemical, surfactant or surface treatments 9 ,10.

In this paper, we present a simple method for the exfoliation of bulk-layered materials and the dispersion of the exfoliated 2D platelets in pure water, which are achieved by merely controlling the temperature of the sonication bath and storage. We tested this approach on several 2D materials, that is, graphene, h-BN, $\mathrm{MoS}_{2}$, $\mathrm{WS}_{2}$ and $\mathrm{MoSe}_{2}$, and observed that, in general, high temperatures favour both exfoliation and dispersion stability. However, the dispersion mechanisms of these materials vary because of their dissimilar surface and physical properties. We envision that such $2 \mathrm{D}$ material solutions can be produced in large volume at low cost, and that they are suitable for printing various patterns without much technical hindrance.

\section{Results}

Exfoliation and dispersion 2D materials in water. We used bulk-layered material powders to synthesize atomically thin platelets in water. The powders were added to deionized water and sonicated for $\sim 60 \mathrm{~h}$ in a bath sonicator (See Methods for materials and sample preparation). To control the bath temperature, we simply adjusted the cold water circulation valve in the bath so as to provide cooling or heating. When cold water circulation was stopped during sonication, the temperature increased to $60^{\circ} \mathrm{C}$ within a few hours because of the dissipation of sonic energy. When cold water was circulated, the temperature was maintained at $30^{\circ} \mathrm{C}$. After sonication, the solutions of the exfoliated $2 \mathrm{D}$ materials were centrifuged at 600 relative centrifugal force to separate the thin flakes. The suspensions of thin $2 \mathrm{D}$ materials in water were stored at either a high temperature $\left(60^{\circ} \mathrm{C}\right)$ or a low temperature $\left(20^{\circ} \mathrm{C}\right)$ for 1 month to determine the temperature dependences of their long-term stabilities. In the synthesis and storage experiments, we used high $\left(60^{\circ} \mathrm{C}\right)$ and low $\left(30^{\circ} \mathrm{C}\right)$ temperatures for exfoliation and high $\left(60^{\circ} \mathrm{C}\right)$ and low $\left(20^{\circ} \mathrm{C}\right)$ temperatures for long-term solution stability studies of the materials.

Figure 1a shows various 2D materials dispersed in water; these dispersions were exfoliated and stored at a high temperature $\left(60^{\circ} \mathrm{C}\right)$. The nanoparticles were found to be suspended stably for longer than 1 month. The colours of the materials in water are as follows: black (graphene), milky-white (h-BN), dark-yellow $\left(\mathrm{MoS}_{2}\right)$, yellow $\left(\mathrm{WS}_{2}\right)$ and light-brown $\left(\mathrm{MoSe}_{2}\right)$. All of the tested materials were found to be stably dispersed by high-temperature sonication and storage. By measuring the light absorption percentage over time with a ultraviolet-visible spectrophotometer, we determined the long-term stabilities of the dispersions of the 2D materials in water (See Methods for ultraviolet-visible measurements). These stability results indicate that the materials sonicated and stored at the high temperature remain stable for one month, as shown in Fig. 1b-d. After 10 days, most of the materials became stable, with little sedimentation afterwards. The graphene sample was found to exhibit the highest stability, with $90 \%$ of the suspension present even after 1 month. We observed that the colour of the graphene solution did not change noticeably even after 1 year. This high stability of our graphene sample is probably due to our optimization of the graphene synthesis process. The concentrations of dispersed flakes from high-temperature sonication and high temperature storage were $0.0065,0.018$ and $0.13 \mathrm{mg} \mathrm{ml}^{-1}$ for graphene, h-BN and $\mathrm{MoS}_{2}$ after one month respectively. Compared to the concentrations of $2 \mathrm{D}$ materials dissolved in organic solvents or surfactants, which are listed in Supplementary Table 1 (See Supplementary Table 2 for the sizes of exfoliated flakes), that of graphene is relatively low, but those of the other materials are comparable $e^{10-15}$. Since the dispersion condition was not optimized for high yield, the dissolution level of these nanoparticles is likely to be improved further as will be discussed later in concluding remarks.

Now, the materials exfoliated at the low temperature $\left(30^{\circ} \mathrm{C}\right)$ were found mostly to be satisfactorily suspended although the overall yield was lower than those sonicated at the higher temperature. However, only the graphene flakes precipitated quickly within three days, which indicates that low-temperaturesonicated graphene is unstable in water (Fig. 1b). To investigate the dependence of the dispersion stability on the storage temperature, the materials exfoliated at 60 and $30^{\circ} \mathrm{C}$ were stored at either 60 or $20^{\circ} \mathrm{C}$. Overall, those stored at the high temperature were found to be more stable than those stored at the low temperature roughly by twice, as shown in Fig. 1b-d (Supplementary Fig. 1 for $\mathrm{WS}_{2}$ and $\mathrm{MoSe}_{2}$ ). According to the DLVO theory on colloid stability ${ }^{16}$, nanoparticles in a solvent interact with each other through van der Waals force, which exerts the attractive and short-range force, and electrostatic force, which exerts the repulsive and long-range force. Between the two forces, the electrostatic force is important to make the suspension stable by preventing aggregation of the particles. Usually, electric double layer is formed around the nanoparticles by ions dissolved in the solvent, and it causes the particles to repulse each other. The thickness of the electric double layer depends on temperature, and increases with temperature. Hence, at higher temperature, the repulsive force also increases and the stability is supposed to be enhanced. Although stability seems to decrease over time in the case of low-temperature storage, we observed that precipitation ceases completely after $\sim 40$ days and that the solutions maintain their same colour for longer than a year (Supplementary Fig. 2). Our exfoliation and solution stability results indicate that solutions of $2 \mathrm{D}$ materials that have been synthesized at the high temperature by sonication in water are quite stable at temperatures above room temperature.

Characterization. Two questions arise from the observed synthesis temperature dependences of the $2 \mathrm{D}$ materials. The first question is why graphene synthesized at the high temperature is stable in water, whereas graphene prepared at the low temperature is not, in contrast to the other $2 \mathrm{D}$ materials. The second question is how the other materials are stably dispersed in water even at the low sonication and storage temperatures. Previous research into graphene dispersion might provide some insight. Although graphene does not readily dissolve in water, graphene oxide is well dispersed because of its surface functional groups ${ }^{10,17-20}$. Hence, it is possible that the 
a
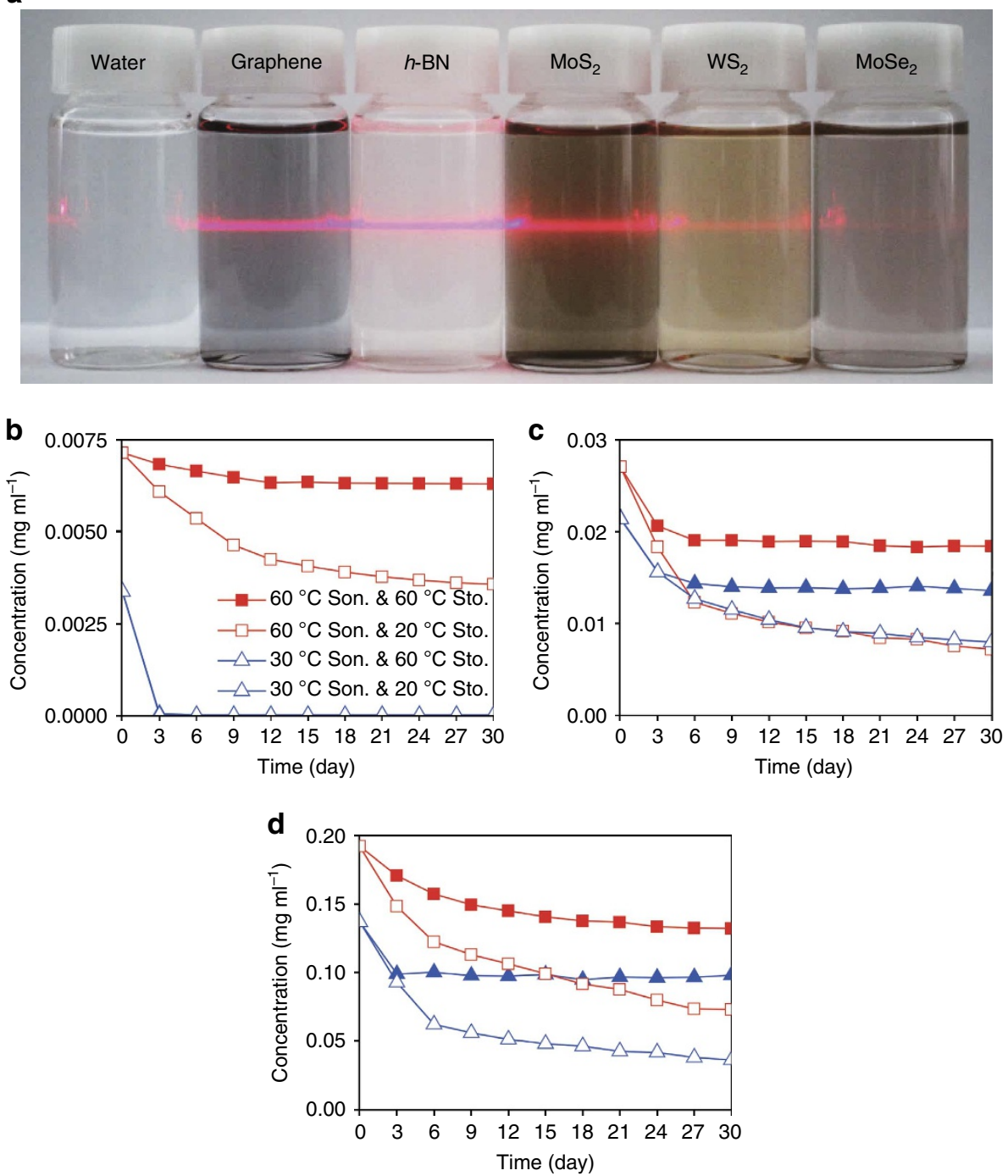

Figure 1 | Temperature-dependent solution stabilities of the 2D materials in water. (a) Photographs of solutions of five 2D materials dispersed in deionized water for one month. The laser light across the solution bottles provides visual assistance because some suspended materials such as $\mathrm{h}$-BN are not clearly visible. The long-term solution stabilities of (b) graphene, (c) h-BN and (d) $\mathrm{MoS}_{2}$ sonicated at the high $\left(60^{\circ} \mathrm{C}\right)$ and low $\left(30^{\circ} \mathrm{C}\right)$ temperature and stored at high $\left(60^{\circ} \mathrm{C}\right)$ and low $\left(20^{\circ} \mathrm{C}\right)$ temperatures. In $(\mathbf{b}-\mathbf{d})$, squares and triangles denote high and low temperature sonication and solid and blank denote high and low temperature storage, respectively. In (b), two types of triangles almost overlap because of fast precipitation.

graphene exfoliated at the high temperature is functionalized, whereas the graphene arising from low-temperature sonication is not. To investigate the functionalization of these materials, we used X-ray photoelectron spectroscopy (XPS) and Fourier transform infrared spectroscopy (FTIR). The XPS spectrum of graphene synthesized with high-temperature sonication shows that it is functionalized with carboxyl and hydroxyl groups (Fig. 2a), which can be identified from peaks at $288.6 \mathrm{eV}$ (carboxyl group) and $286.2 \mathrm{eV}$ (hydroxyl group). In contrast, the spectrum of graphene prepared with low-temperature sonication indicates that only a negligible number of such functional groups are present (Supplementary Fig. 3a). FTIR spectra of graphene samples synthesized with high- and low-temperature sonication are displayed in Fig. 3. That of the graphene sample from high-temperature sonication shows appearance of new peaks at 1,024 and $1,094 \mathrm{~cm}^{-1}$, corresponding to $\mathrm{C}-\mathrm{O}$ stretches in hydroxyl groups, and at $1,260 \mathrm{~cm}^{-1}$, corresponding to a $\mathrm{C}-\mathrm{O}$ stretch in carboxyl or epoxy group, while they were not observed in the FTIR spectra of graphite and graphene samples from low-temperature sonication ${ }^{21}$. It clearly confirms that high-temperature sonication leads to successful functionalization of oxygen-containing hydrophilic groups on graphene network. In contrast, there is no evidence of functionalization in the XPS and FTIR data for h-BN and $\mathrm{MoS}_{2}$ (Fig. 2a and Supplementary Fig. 4).

To determine whether these functional groups of graphene are present on the surface, as in graphene oxide, or on the edges, we performed Raman spectroscopy on a single-layer graphene flake obtained with high-temperature sonication. The intensity of the D-peak in the Raman spectrum of the central area is negligible, which indicates the bare existence of functionalization or defects (Fig. 2b). The peaks in the Raman spectrum of bulk graphite, which is the raw material used in the synthesis of the graphene sample, have similar shapes, except for that of the D-peak; this difference is due to the layer edges (Supplementary Fig. 5a). The Raman spectra of the other materials indicate that they are not damaged structurally by the sonication process (Fig. 2b and Supplementary Fig. 5b,c). Also they prove that the materials were exfoliated down to monolayer from high $2 \mathrm{D} / \mathrm{G}$ peak ratio in graphene spectrum ${ }^{22}$, which is the clear evidence of single-layer 


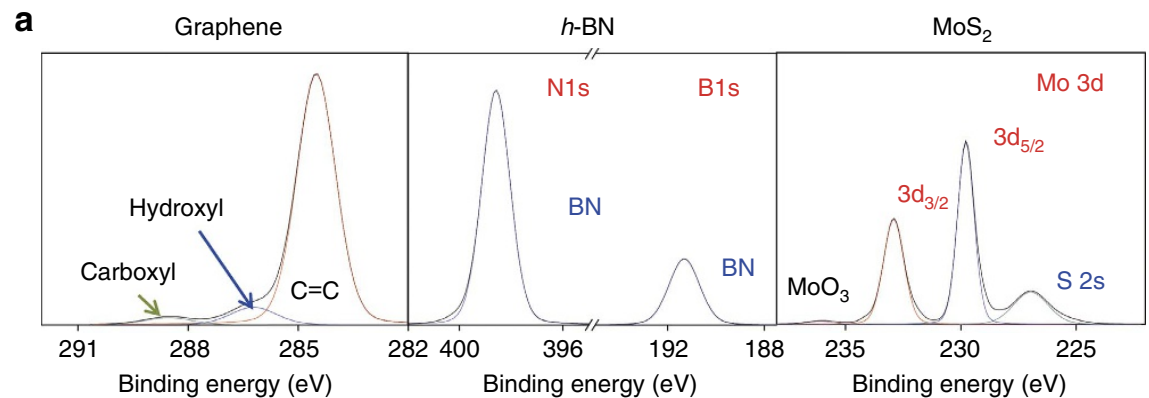

b

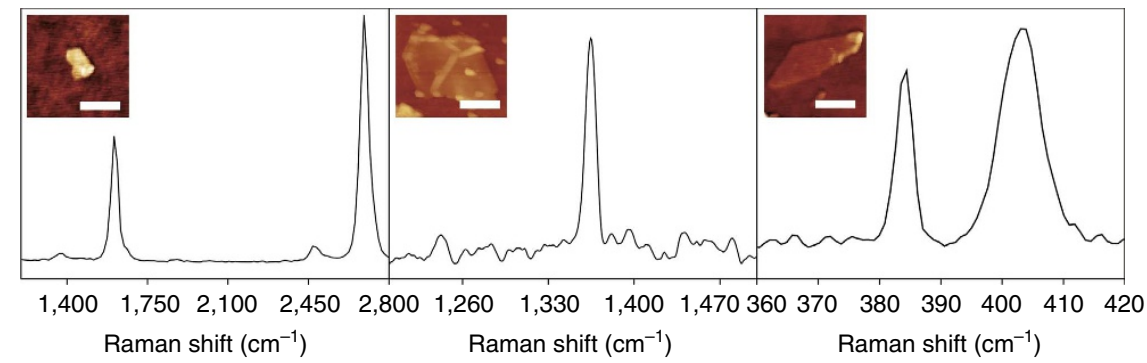

C

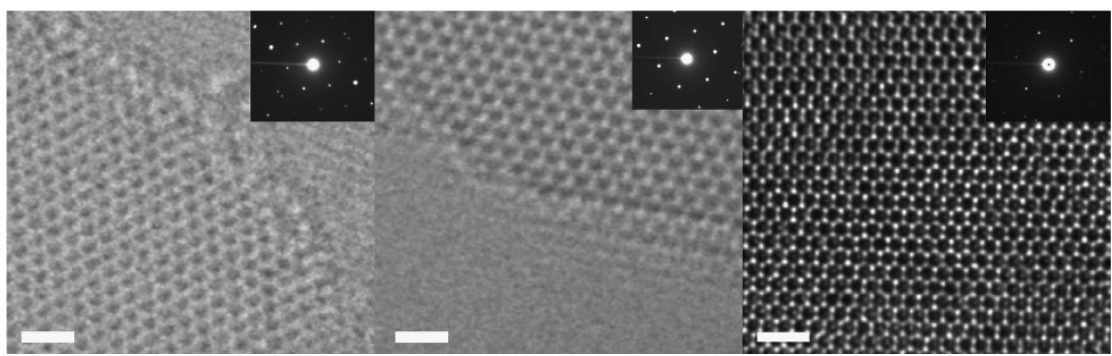

Figure 2 | Analyses of the chemical compositions of the synthesized materials. (a) XPS, (b) Raman spectroscopy, and (c) high-resolution TEM. The scale bars in (c) are $1 \mathrm{~nm}$.

graphene, and the gap of $18.5 \mathrm{~cm}^{-1}$ between $\mathrm{E}_{2 \mathrm{~g}}$ (ref. 1) peak and $\mathrm{A}_{1 \mathrm{~g}}$ peak in $\mathrm{MoS}_{2}$ spectrum, which is also a solid proof of singlelayer $\mathrm{MoS}_{2}$ (ref. 23). We also could confirm the exfoliation of monolayer $\mathrm{MoS}_{2}$ through photoluminescence (PL) spectroscopy, since monolayer $\mathrm{MoS}_{2}$ has distinct peak positions and high intensity at around 1.82 and $1.97 \mathrm{eV}$ compared with thicker layers $^{24}$. (See Supplementary Fig. 6) For h-BN, we could hardly identify single-layer due to the small size and poor optical contrast of the flakes (in average $200 \mathrm{~nm})^{25}$, thus we identified samples of a few layers optically and got the Raman data. However, thinner layers such as monolayer could be identified through transmission electron microscopy (TEM) as explained below.

To assess the quality and distribution of thickness of the exfoliated flakes, we collected high-resolution TEM images, as shown in Fig. 2c. The in-plane crystalline structures of the materials are well ordered without defects. These images reveal that sonication results in little damage to or chemical reaction on the surfaces of these materials. On the graphene edge, the atoms are not discernible, which is an indirect indication of edge functionalization. In contrast, the edge atoms of h-BN are well ordered without signs of functionalization. A previous study of the water dispersion of h-BN speculated that good dispersion is due to edge functionalization despite its hydrophobic surface ${ }^{25}$. However, our TEM observations show that h-BN does not dissolve because of functionalization. In addition, a previous theoretical study provides evidence that h-BN is hardly oxidized due to the thermodynamic instability of its oxide ${ }^{26}$. The TEM image of $\mathrm{MoS}_{2}$ also shows that its crystal structure is not damaged or oxidized by sonication, at least in the centre. Our TEM observations also confirm the results obtained with optical spectroscopies for the structures and functionalization of graphene, h-BN, and $\mathrm{MoS}_{2}$. We also determined the thickness distributions of the suspended flakes by using low-resolution TEM imaging, as shown in Supplementary Fig. 7. Overall, the dispersed materials consist mostly of 2-3 layers and the portion of monolayer was $10-20 \%$. The flake size is $\sim 200-300 \mathrm{~nm}$.

Our spectroscopy and TEM results indicate that graphene sonicated at the high temperature is functionalized only on its edges and that these functional groups enhance the solubility of graphene in water. This conclusion raises another question: how is graphene functionalized when sonicated at the high temperature. In an ultrasonic bath, cavitation occurs because of abrupt pressure fluctuations. Cavitation is a phenomenon in which bubbles are formed in water and collapse within milliseconds. Bubble collapse induces a quite high local temperature of $\sim 5,000^{\circ} \mathrm{C}$ and a high local pressure of tens of thousands of bars ${ }^{27}$. Water can decompose into chemically volatile $\mathrm{H}^{+}$and $\mathrm{OH}^{-}$ions at such conditions, so cavitation can provide sufficient energy to break bulk-layered materials and induce the chemical reactions of such materials with water. This type of sonochemical reaction (or sonochemistry) has been used in the synthesis of diverse nanomaterials ${ }^{28}$. The graphene edge has dangling carbon atoms, so more easily reacts with radicals or ions than does the in-plane area ${ }^{29}$. In cavitation by ultrasonic agitation, usually the sonic pressure amplitude should be greater than the difference between the atmospheric pressure and the 


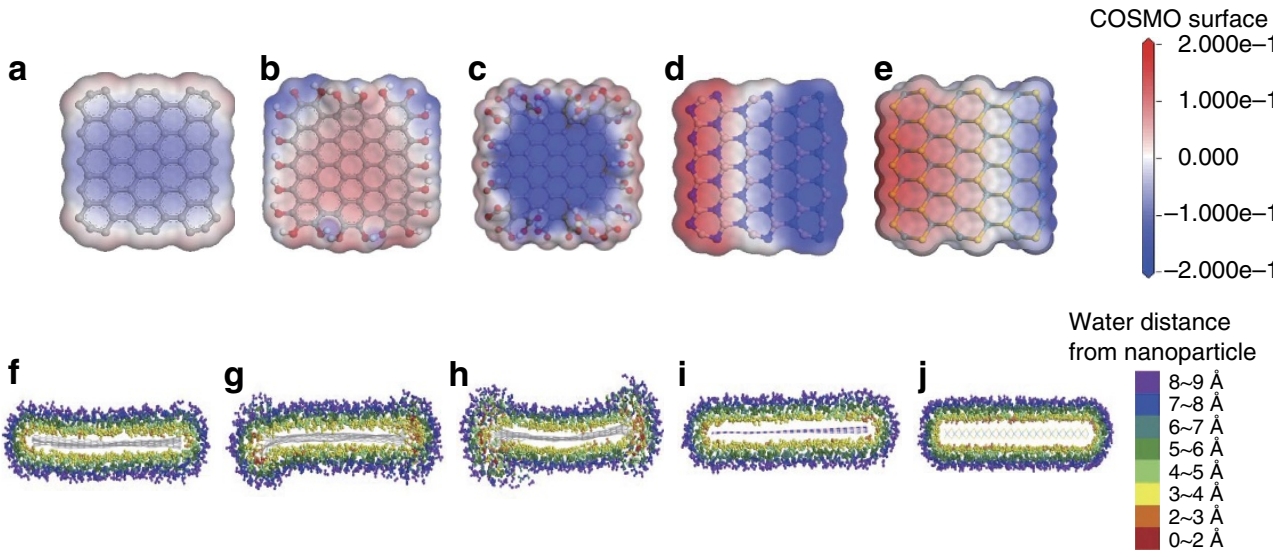

Figure 3 | Molecular simulation results of solubility of 2D materials in water. Charge density of water around (a) pristine graphene, (b) -OH functionalized graphene, (c) $-\mathrm{COOH}$ functionalized graphene, (d) h-BN, and (e) $\mathrm{MoS}_{2}$. The isosurface is constructed from the COSMO-solvation model. The change of surface charge is much larger in polar nanoparticles. Water distributions around (f) pristine graphene, ( $(\mathbf{g})-\mathrm{OH}$ functionalized graphene, (h) $-\mathrm{COOH}$ functionalized graphene, (i) h-BN, and (j) $\mathrm{MoS}_{2}$

boiling pressure at a given temperature; this difference is $\sim 1 \mathrm{~atm}$ under ambient conditions. When sound propagates in matter, the pressure is attenuated by energy absorption. In water, the attenuation level is higher at lower temperatures, which means that the sonic pressure is lower at lower temperatures because cold water can absorb the heat dissipated from the sonic energy easily ${ }^{30,31}$.We measured the sonic pressure in the bath at two different temperatures: $60^{\circ} \mathrm{C}$ and $30^{\circ} \mathrm{C}$. The sonic pressure at $60^{\circ} \mathrm{C}$ was found to fluctuate by as much as $2.4 \mathrm{~atm}$, which can easily induce cavitation, whereas at $30^{\circ} \mathrm{C}$ the sonic pressure fluctuates by as much as $0.46 \mathrm{~atm}$ (Supplementary Fig. 8). These temperature-dependent sonic pressure measurements suggest that the threshold temperature for the active sonochemical functionalization of graphene would be around $40^{\circ} \mathrm{C}$, above which graphene can be dispersed with long-term stability.

For the 2D materials other than graphene, other factors must be present that induce their dissolution in water regardless of the synthesis temperature. In contrast to graphene, h-BN, $\mathrm{MoS}_{2}$, $\mathrm{WS}_{2}$ and $\mathrm{MoSe}_{2}$ dispersions remain stable when dispersed via high- and low-temperature sonication, which indicates that edge functionalization is not the cause of the high water solubility of these materials. These materials are electrically less conductive than graphene, so an electrical double layer can form on their surface and thus the materials can be readily suspended in water without agglomeration or sedimentation. This hypothesis can be assessed by performing zeta-potential measurements because nanoparticle solution stability is closely related to the electrical double layers on nanoparticle surfaces. In general, dispersed materials with zeta-potential values near or $<-30 \mathrm{mV}$ are stable ${ }^{32-34}$. The zeta-potential values of the h-BN, $\mathrm{MoS}_{2}, \mathrm{WS}_{2}$ and $\mathrm{MoSe}_{2}$ dispersion samples are all $\sim-30 \mathrm{mV}$, irrespective of the sonication temperature (Supplementary Fig. 9). The zeta-potential of the high-temperature-sonicated graphene sample is similar. However, the zeta-potential of the graphene sample sonicated at the low temperature is $-13 \mathrm{mV}$ and this sample exhibits low solution stability. Therefore, the zeta-potential results support our argument for a temperaturedependent dispersion mechanism in water.

The $\mathrm{pH}$ of a dispersion solution can change during sonication because of sonochemical reactions, and this change might affect solubility. In the case of graphene, h-BN, and $\mathrm{MoSe}_{2}$, the $\mathrm{pH}$ varies little; hence, the $\mathrm{pH}$ does not appear to influence their solubility. However, the $\mathrm{MoS}_{2}$ and $\mathrm{WS}_{2}$ solutions became acidic after sonication (Supplementary Fig. 10). To investigate the effects of $\mathrm{pH}$ on solubility, we measured the $\mathrm{pH}$ of aqueous solutions of the raw material powders and found that they are similar to the $\mathrm{pH}$ values of the dispersed $2 \mathrm{D}$ material solutions. However, the particles in powder solutions mostly precipitate regardless of temperature, in contrast to the sonicated solutions; these results indicate that $\mathrm{pH}$ is not a major contributor to the high solubility of the $2 \mathrm{D}$ materials. To show that the $2 \mathrm{D}$ materials are dispersed in water due to exfoliation, we prepared control samples of graphite, which were just stirred at 60 and $30^{\circ} \mathrm{C}$ and compared with the sonicated samples as shown in Supplementary Fig. 11. The simply stirred graphite particles of both temperatures precipitated quickly within several hours and faster than sonicated samples.

Numerical calculation of water-nanoparticle interaction. In order to further investigate the solvation states of these materials, we performed molecular dynamics (MD) simulations and $a b$ initio calculations for pristine graphene, functionalized graphene (hydroxyl $(-\mathrm{OH})$ and carboxyl $(-\mathrm{COOH})$ groups), h-BN and $\mathrm{MoS}_{2}$ (See Methods for calculation methods). Hydroxyland carboxyl-functional groups on graphene materials are chosen as possible functionalities from above XPS and FTIR characterization. Pristine graphene has a weakly positive charge due to dangling carbon atoms, and carboxyl-functionalized graphene has a stronger positive charge due to the electronwithdrawing carboxyl groups, whereas the electron-donating hydroxyl groups render graphene flakes negatively charged. On the other hand, h-BN has an alternating charge distribution and exhibits strong polarity across the boron and nitrogen termination edges and $\mathrm{MoS}_{2}$ shows the negative charge on the surface by $\mathrm{S}$ atoms and counter charges inside by Mo atoms, which exhibit moderate polarity (Supplementary Fig. 12). Due to the surface charges of the materials, water molecules, which are polar, are attracted and cause charge distributions in water as shown in Fig. 3a-e. The numerical calculation results of interaction between the $2 \mathrm{D}$ materials and water show that the solvation level is highest for carboxyl-functionalized graphene, the intermediate for hydroxyl-functionalized graphene, h-BN and $\mathrm{MoS}_{2}$, and the lowest for pristine graphene (See Methods for calculation results). Furthermore, we investigated the distribution of water molecules around the nanoparticles (Fig. $3 \mathrm{f}-\mathrm{j}$ and Supplementary Fig. 13). Water molecules within $\sim 6 \sim 8 \AA$ of the nanoparticles were found to reorient in charged layers so as to 
neutralize the charged nanoparticles, which means that edge effects due to functional groups or $\mathrm{B}$ and $\mathrm{N}$, or Mo and $\mathrm{S}$ are dominant. This particular orientation of the water molecules leads to stronger interactions with the nanoparticles; the strengths of these interactions are estimated to be greater than that of the water-water interaction. Note that the overall attractive interaction energy is due to weak van der Waals interactions and strong electrostatic interactions. It has been found that a charge distribution or charge polarity on the surfaces of materials enhances their interactions with water molecules, hence functionalized graphene, h-BN and $\mathrm{MoS}_{2}$ are more water-soluble and stable than pristine graphene, which is in good agreement with our experimental observations.

Printing application of the solutions. Our water-based synthesis method is facile and will be potentially cost-effective, and the materials are well dispersed; thus, diverse applications should be possible. We performed inkjet printing with the dispersed 2D material solutions since the as-prepared solutions can be used directly as inks. First, we used the nozzle printing method applying a hydraulic pressure to the nozzle to draw lines on $\mathrm{Si}$ and $\mathrm{Si} / \mathrm{SiO}_{2}$ with graphene, h-BN, and $\mathrm{MoS}_{2}$ dissolved in pure water as shown in Fig. $4 \mathrm{a}-\mathrm{c}$ (See Methods for printing methods). Since the viscosity of water is quite low, printing with inks based on pure water is extremely challenging. We increased the concentration of the $2 \mathrm{D}$ materials in the solution by evaporating major portion of water and controlled the substrate temperature at $50{ }^{\circ} \mathrm{C}$ to achieve successful printing with water solutions. There is an intrinsic limitation on the reduction in line width that can be achieved with water-based inks due to the low viscosity of water, so we mixed a polymer poly (ethylene oxide) (PEO) with the water solutions of the $2 \mathrm{D}$ materials to increase their viscosity and performed electrohydrodynamic (EHD) printing by applying an electric field between the nozzle and the substrate, as shown in Fig. 4d-f (see Methods for printing details). With this approach, we were able to draw lines with a width of $20 \mu \mathrm{m}$, which can be further reduced by replacing the nozzle with one with a smaller diameter and optimization. We could also print other patterns such as letters and mesh on the hard substrates of $\mathrm{SiO} 2 / \mathrm{Si}$ and $\mathrm{Si}$ and, and a mesh pattern on a flexible substrate as shown in Supplementary Fig. 14.

Graphene is a prominent conductor, so we characterized the electrical properties of the graphene printed lines and films as shown in Fig. 4g,h and Supplementary Fig. 15. The PEO-graphene composite was found to exhibit a conductivity of $6 \times 10^{-5} \mathrm{~S} \mathrm{~m}^{-1}$, which is comparable to that of a previous graphene-contained composite ${ }^{35}$. Unlike the graphene composite, in which the conductive graphene flakes are separated by polymer, the flakes are directly connected with each other in a pure-graphene electrode, hence the conductivity was much higher, with a value of $50 \mathrm{~S} \mathrm{~m}^{-1}$. Since the graphene flakes would be poorly stacked due to the rapid printing and drying process in this experiment, the electrical property appears relatively low compared with graphene or reduced graphene oxide samples synthesized by other methods ${ }^{11,12,36,37}$. Hence, we fabricated graphene films by filtering the solution through an anodisc filter and found the electrical conductivity to be $440 \mathrm{~S} \mathrm{~m}^{-1}$, which is about one order higher than the printed film (See methods and Supplementary Fig. 15). Because this film was made by a slower process, the stacking would be more ordered. Therefore, we judge that there is a room for further enhancement of electrical properties by improving the film fabrication process. This level of conductivity is quite encouraging
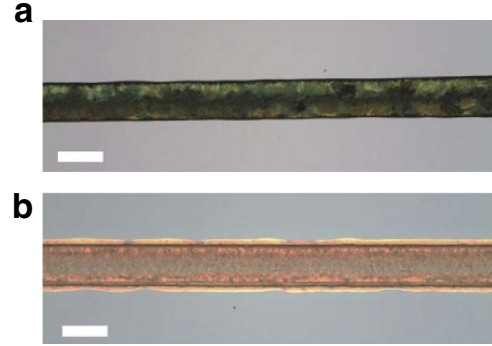

C

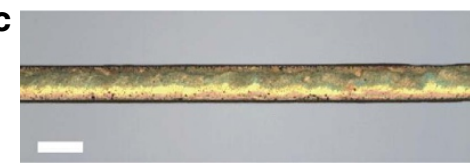

d

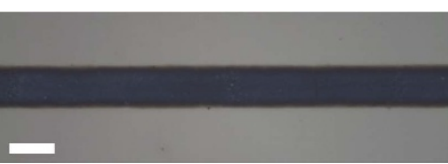

e

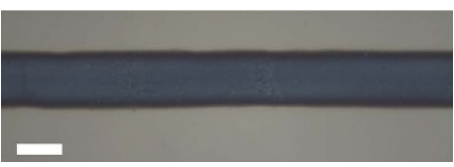

f

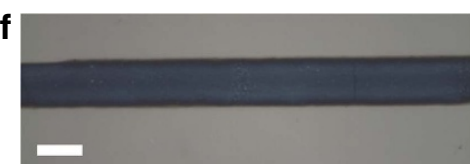

g

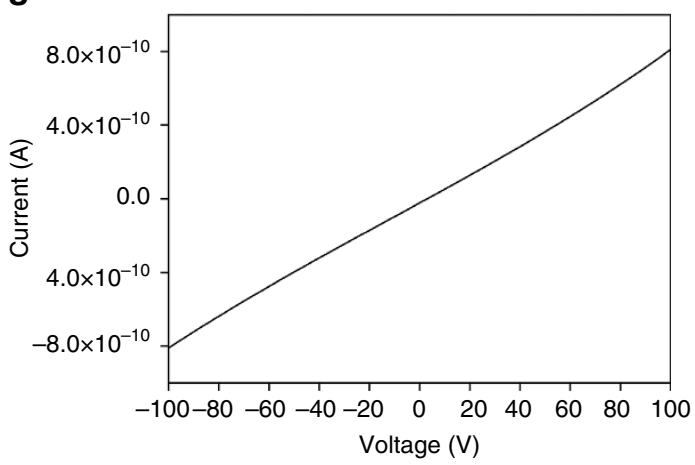

h

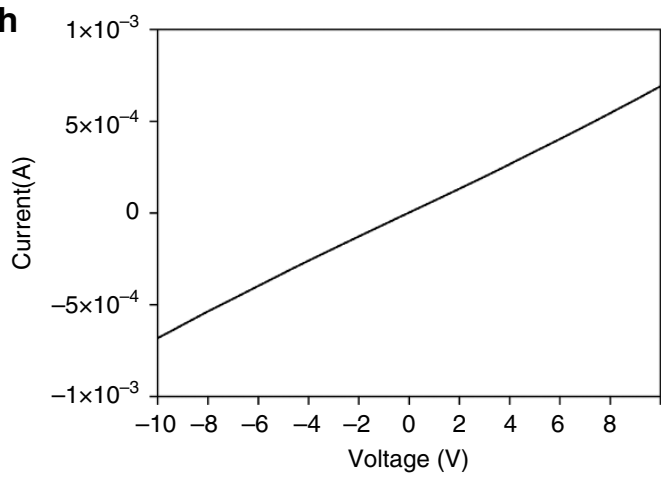

Figure 4 | Inkjet printing results with 2D material solutions. Lines printed with pure-water inks of (a) graphene, (b) h-BN, and (c) MoS 2 , and mixed PEOwater inks of (d) graphene, (e) h-BN, and (f) $\mathrm{MoS}_{2}$. Electrical conductivity measurements for (g) a pure-graphene electrode and (h) a mixed PEO-water graphene electrode. The scale bars in (a-c) and (d-f) are 300 and $20 \mu \mathrm{m}$. 
that the graphene films would be able to be used as an electrode for electric devices. The printing results indicate that the waterdispersed 2D material inks are promising for printed electronics, with no significant hindrances to practical applications.

\section{Discussion}

The results of our dispersion experiments with the 2D materials show that sonication at a high temperature results in the synthesis of 2D materials with good dispersion stabilities in water. This ability to use pure water to exfoliate and disperse $2 \mathrm{D}$ materials will enable not only cost-effective industrial and commercial applications but also facilitate research that requires water-based experiments. The heat required to raise the sonication bath temperature originates from the dissipation of sonic energy, so no additional heat energy is required for the production of the exfoliated materials. To date, ultrasonic cavitation has only been used for the physical exfoliation of $2 \mathrm{D}$ materials via the breaking of bulk lamellar materials into thinner flakes ${ }^{15}$. However, in this study, we demonstrated the first synthesis of water-soluble graphene by performing a sonochemical reaction through temperature-controlled sonication. Since cavitation is the key factor to the $2 \mathrm{D}$ materials dispersion in water, the particle dissolution yield can be improved by heating the water more and lowering the pressure, which enhances the level of cavitation. Our results and findings further the development of large-volume and economical synthesis methods for $2 \mathrm{D}$ materials using sonochemistry. We have achieved inkjet printing with waterdispersed 2D material inks, which thus have significant potential for real-life applications.

\section{Methods}

Materials and sample preparation. For sample preparation, we purchased graphite and $\mathrm{MoSe}_{2}$ powders from Alfa Aesar. h-BN powder was purchased from Momentive. $\mathrm{MoS}_{2}$ and $\mathrm{WS}_{2}$ were purchased from Sigma Aldrich.

To exfoliate and disperse $2 \mathrm{D}$ materials in water from the source powders, we used a bath sonicator, manufactured by Onejon Ultrasonic in Korea. Each material $(20 \mathrm{mg})$ was sonicated in deionized water $(200 \mathrm{ml})$ for $60 \mathrm{~h}$. The operation power was $20 \mathrm{~W}$ and frequency was $40 \mathrm{kHz}$. In addition, the cooling water circulation equipment was built in the sonicator to control the sonication bath temperature. The resulting solution was centrifuged at 600 relative centrifugal force for $30 \mathrm{~min}$ using a high speed centrifuge (Supra 25K, HANIL SCIENCE INDUSTRIAL). The final solution was kept either in an atmospheric condition $\left(20^{\circ} \mathrm{C}\right)$ or in an oven $\left(60^{\circ} \mathrm{C}\right)$.

Dispersion stability measurement. We first measured the concentration of the $2 \mathrm{D}$ materials right after sonication and centrifugation by weighing the mass of the dried flakes. When measuring the mass, we prepare two identical solution samples, so that one can be used for mass measurement, and the other one for concentration change measurement. We observed the concentration change (or stability) of the dispersions using the ultraviolet-VIS-NIR spectrophotometer (UV-3600, SHIMADZU) to confirm how long the flakes are stably suspended in water. We put the solutions in standard $1-\mathrm{cm}$ path quartz cell and measured the absorbance for 1 month with the interval of 3 days. The stability represents the absorbance at $650 \mathrm{~nm}$ normalized by the first measured value. The absorbance at $650 \mathrm{~nm}$ has been normally used to compare the stability of graphene in a dispersion study ${ }^{15,38,39}$. The stability of $\mathrm{MoSe}_{2}$ and $\mathrm{WS}_{2}$ in water is shown in Supplementary Fig. 2 (See the main paper for the stability of graphene, h-BN and $\mathrm{MoS}_{2}$ ). The $\mathrm{WS}_{2}$ stored at high temperature is considerably stable in water for 1 month while the stability at low temperature a little bit lower over the time. We can know the stability of $\mathrm{WS}_{2}$ suspended in water is dependent on the storage temperature, like graphene, h-BN and $\mathrm{MoS}_{2}$ dispersions. However, the solubility of $\mathrm{MoSe}_{2}$ dispersed in water is high at both high and low storage temperature. It is worth noting that once $\mathrm{MoSe}_{2}$ is exfoliated in water, the dispersion can be very stable regardless of the storage temperature.

XPS measurement. XPS analysis was performed to investigate the functional groups of graphene, h-BN and $\mathrm{MoS}_{2}$ flakes exfoliated at each temperature. Each suspension of graphene, h-BN and $\mathrm{MoS}_{2}$ was dropped onto a quartz substrate using a pipette until flakes totally covered the substrate. The thin films on the quartz substrate were annealed at $160^{\circ} \mathrm{C}$ under Ar gas flow to dry up the water moisture. We analysed the samples using K-Alpha X-ray Photoelectron Spectrometer system with monochromated $\mathrm{Al} \mathrm{X}$-ray sources. The survey scan was $-50 \mathrm{eV}$, and the step size was $0.1 \mathrm{eV}$. Flood gun was used for charge compensation.
Supplementary Fig. 3a shows the XPS data of graphene sonicated at low temperature. The spectrum does not have functional groups, which are shown in high-temperature sonicated graphene. Supplementary Fig. $3 \mathrm{~b}$ shows the XPS data of the $\mathrm{MoS}_{2}$ powder. There are some peaks representing $\mathrm{MoS}_{2}$, such as $3 \mathrm{~d} 3 / 2$ and $3 \mathrm{~d} 5 / 2$ peaks, related to $\mathrm{Mo}$ and $\mathrm{S} 2 \mathrm{~s}$ peak due to $\mathrm{S}$ bonded to $\mathrm{Mo} . \mathrm{MoO}_{3}$ peak also exists, and this peak means the oxide bonded to Mo. There is almost no difference between the $\mathrm{MoS}_{2}$ flakes and $\mathrm{MoS}_{2}$ powder. It is noted that the $\mathrm{MoO}_{3}$ peak is observed at both spectra, and the atomic per cent and ratio of $\mathrm{MoS}_{2}$ flakes are similar to those of the $\mathrm{MoS}_{2}$ powder. From the $\mathrm{MoO}_{3}$ peak and the atomic per cent of $\mathrm{O}$, we can conclude that the $\mathrm{MoS}_{2}$ flakes in water were not functionalized, as opposed to graphene (see the main manuscript for the graphene). Also, the h-BN flakes were not functionalized during sonication process, as shown in Supplementary Fig. 3c.

FTIR spectroscopy. FIIR analysis was performed to investigate functional groups of graphene h-BN, and $\mathrm{MoS}_{2}$ flakes exfoliated at each temperature. Each suspension was filtered through an anodisc membrane by using an aspirator, and then the filtered film was transferred to a silicon substrate. The thin film on the substrate was annealed at $160^{\circ} \mathrm{C}$ under Ar gas flow to dry up the water moisture. We analysed the h-BN and $\mathrm{MoS}_{2}$ samples using Bruker IFS-66/S Fourier Transform Infrared Spectroscopy system. FTIR spectra of graphene and graphite samples were obtained from $\mathrm{KBr}$ pellets using an FTIR vacuum spectrometer (Bruker VERTEX 80V, Bruker, Germany) with 64 scans.

Raman spectroscopy. We performed the Raman spectroscopy to confirm the quality of the nanosheets of graphene, h-BN, $\mathrm{MoS}_{2}$ exfoliated in water and to compare with the raw materials. Films on silicon oxide substrate for the measurement were prepared as the films for FTIR spectroscopy except for the substrate material. The excitation wavelength was $532 \mathrm{~nm}$. Supplementary Fig. 4 Raman shows the spectra of the powders of raw materials, which are similar to the spectra of the water-exfoliated flakes. From Raman spectra of the four materials, we could not find oxide peaks, which reveals that they were not oxidized during the synthesis $40-42$.

PL spectroscopy. We performed the photoluminescence analysis to confirm the exfoliation of $\mathrm{MoS}_{2}$ to single and few layers. The $\mathrm{MoS}_{2}$ flakes were deposited on a silicon substrate. The excitation wavelength was $532 \mathrm{~nm}$. The strong intensity of peaks at $1.82 \mathrm{eV}(680 \mathrm{~nm})$ and $1.97 \mathrm{eV}(630 \mathrm{~nm})$ confirms the existence of monolayers among the flakes, and those peaks are contrasted with bilayer and trilayer flakes shown in Supplementary Fig. 5 (ref. 43).

Low-resolution TEM image and distribution of sheet thickness. The nanosheets were further analysed using a TEM. A drop of dispersion was casted on holy carbon grid (400 mesh) for the measurement. Low-resolution TEM imaging was conducted using FE-TEM manufactured by JEOL Ltd. with the acceleration voltage of $200 \mathrm{kV}$. The upper row of Supplementary Fig. 5 shows the typical images of 2D materials. We can observe monolayer, folded mono- or bi- layer, and few layers. In addition, thickness distributions of sheets were plotted through the investigation in TEM results as shown in the lower row of Supplementary Fig. 5. Overall, about $10 \%$ of the flakes were monolayer, and about $87 \%$ of observed flakes were thinner than 5 layers. In fact, we can know that the dispersions prepared by present method contain very thin nanosheets.

Atomic resolution TEM imaging. Specimens were analyzed using an aberrationcorrected FEI Titan Cubed TEM (FEI Titan ${ }^{3}$ G2 60-300), which was operated at $80 \mathrm{kV}$ acceleration voltage with a monochromator.

Measurement of the sonic pressure. The beaker was placed in the centre of the bath sonicator. The beaker was filled with deionized water without particles in order to prevent contamination of the probe and the artifacts from the particles. The sonic wave was measured at the centre of the beaker for $5 \mathrm{~ms}$ with a hydrophone (Gearing \& Watson D/140) to obtain sonic pressure data.

Supplementary Fig. 6 shows temporal waveforms and spectra for the two different temperatures of the sonication bath. The main frequency peak appears at around $37 \mathrm{kHz}$. The frequency peaks can vary depending on the temperature and the dissolved particles. The measured sonic pressure values at high $\left(60^{\circ} \mathrm{C}\right)$ and low $\left(30^{\circ} \mathrm{C}\right)$ temperatures are $237,000 \mathrm{~Pa}$ and $46,000 \mathrm{~Pa}$.

Electrokinetic potential of 2D flakes in water. We measured zeta potential of the centrifuged dispersions using zetasizer (NANO ZS, Malvern instruments). Usually, when the zeta potential is below $-30 \mathrm{mV}$, the dispersion quality of flakes in water is quite excellent ${ }^{10}$. The graphene sonicated at high temperature was below- $30 \mathrm{mV}$. Just considering the zeta potential value, graphene synthesized by our method appears better compared with a reduced graphene oxide ${ }^{10}$. However, the graphene prepared at low temperature has poor zeta potential. For the other materials, the zeta potential was, in general, lower with high temperature sonication. 
pH measurement. The hydrogen exponent of the aqueous solutions was measured to confirm the state of the solution using $\mathrm{pH}$ metre (E-sweep, Seiko Instruments Inc.). Supplementary Fig. 10 shows the $\mathrm{pH}$ values of all materials dispersed in water.

Molecular modelling and simulation. We constructed five platelet-nanoparticle models, which are pristine graphene, hydroxyl-functionalized $(-\mathrm{OH})$ graphene and carboxyl-functionalized $(-\mathrm{COOH})$ graphene with basal planes of the side length about $5 \mathrm{~nm}, \mathrm{~h}-\mathrm{BN}$, and $\mathrm{MoS}_{2}$ with the edge types of Mo-edge with $0 \% \mathrm{~S}$ atoms and S-edge with $100 \% \mathrm{~S}$ atoms. Note that we fully functionalized edges of graphene with functional group for each simulation to observe clearer effects. Mulliken-based partial charges of the models were estimated by the population analysis method incorporated in $\mathrm{DMol}^{3}$ (refs 44,45), which is able to perform the density function theory (DFT) calculation, through investigating nanoribbon models of pristine graphene, functionalized graphenes, h-BN, and $\mathrm{MoS}_{2}$. Note that partial charges of hydrogen $(+0.41 \mathrm{e})$ and oxygen $(-0.82 \mathrm{e})$ in water molecule were used. Supplementary Fig. 12 shows the results of charge distributions of the nanoparticles. For investigating charge distribution of water molecules surrounding nanoparticles, we have performed MD simulations of each nanoparticle submerged in water with a box size of $10 \times 10 \times 3.6 \mathrm{~nm}^{3}$. We used different potential parameters for each material to describe inter- and intra-molecular interaction. The COMPASS forcefield for pristine and functionalized graphenes, DREIDING forcefield for h-BN and fitted forcefield originated from works by Varshney et al ${ }^{46}$ and Morita et al. ${ }^{47}$ for $\mathrm{MoS}_{2}$ were used, respectively (Supplementary Table 3). MD simulations have been performed with the isothermal-isobaric ensemble (that is, NPT, where $\mathrm{N}$ is the number of molecules, $\mathrm{P}$ is pressure, and $\mathrm{T}$ is temperature) for 50 ps with a time step of $1 \mathrm{fs}$ followed by the canonical ensemble (that is, NVT, V is volume) for over $200 \mathrm{ps}$ with the same time step at $1 \mathrm{~atm}$ and $60^{\circ} \mathrm{C}$. For monitoring temperature and pressure, Berendsen thermostat and barostat were used, respectively. For the detailed analysis, we partitioned the volume by isosurface shell contouring the equilibrated nanoparticle in $3 \mathrm{D}$ at an interval of $0.5 \AA$ and averaged charges of hydrogen and oxygen atoms over the shell volume. In the same context, the non-bonding interaction energy (that is, van der Waals and electrostatic energy) between water molecules in each shell and nanoparticle has been calculated. The results are shown in Supplementary Fig. 13.

The solvation energy is calculated by the interaction of nanoparticles with implicit water molecules. As shown from Supplementary Fig. 12, we obtained the gas phase geometries of nanoparticles by the DFT calculation using $\mathrm{DMol}^{3}$ (refs 44,45 ) with the generalized gradient approximation with the Perdew-BurkeErnzerhof functional ${ }^{48}$. The effect of implicit water environment is included by using the COSMO(Conductor-like screening model $)^{49}$ scheme. Unrestricted spin-polarized calculations were performed with basis set of DNP 4.4 level, and the SCF convergence criterion was set to be $<1.0 \times 10^{-6}$ ha. The calculated solvation energies for graphene, graphene with hydroxyl group, graphene with carboxyl group, h-BN, and $\mathrm{MoS}_{2}$ are $-23.45,-66.46,-213.4,-129.6$ and $-130.3 \mathrm{kcal} \mathrm{mol}^{-1}$, respectively.

The Flory-Huggins parameter $\chi$ measures the interaction related to mixing of solvent and solute. As $\chi$ decreases, it can be considered that solute is more soluble in solvent. To obtain $\chi$, the solubility parameter $\delta$ of nanoparticle has been estimated from MD simulation, of which the system contains 200 nanoparticles. We chose to use the Hansen solubility parameter, which is known to be good for predicting miscibility of polar molecules. The NPT MD simulation has been performed for the system to be at an equilibrated density at $298 \mathrm{~K}$ and subsequently, the NVT MD simulation has been performed for further equilibration. Dispersion and electrostatic solubility parameters were obtained from resulting configurations of the NVT MD simulation. Since the Hansen solubility parameter is composed of dispersion $\delta_{\mathrm{d}}$, polar $\delta_{\mathrm{p}}$ and hydrogen bonding $\delta_{\mathrm{hb}}$ terms by the following relation ${ }^{50}$,

$$
\delta_{\text {Hansen }}=\sqrt{\delta_{\mathrm{d}}^{2}+\delta_{\mathrm{p}}^{2}+\delta_{\mathrm{hb}}^{2}}
$$

the three solubility parameters have been obtained with the help from the DFT calculation by the COSMO scheme. In particular, for the polar solubility parameter, the following equation was applied ${ }^{51}$

$$
\delta_{\mathrm{p}}=t\left(\frac{1.29}{\sqrt{V_{\text {cosm }}}}-1.10\right)
$$

where $t$ is dipole moment in the unit of Debye and $V_{\text {cosm }}$ is COSMO volume $\left(\mathrm{nm}^{3}\right)$. From these solubility parameters, the Flory-Huggins parameter was finally calculated by the following equation ${ }^{50}$

$$
\chi_{\mathrm{ij}}=\frac{v_{0}}{k_{B} T}\left[\left(\delta_{\mathrm{d}_{\mathrm{i}}}-\delta_{\mathrm{d}_{\mathrm{j}}}\right)^{2}+0.25\left(\delta_{\mathrm{p}_{\mathrm{i}}}-\delta_{\mathrm{p}_{\mathrm{j}}}\right)^{2}+0.25\left(\delta_{\mathrm{hb}_{\mathrm{i}}}-\delta_{\mathrm{hb}_{\mathrm{j}}}\right)^{2}\right]
$$

where the subscripts $i$ and $j$ represent each nanoparticle and water, respectively, and $v_{0}$ is the solvent molecular volume, which is $30 \AA^{3}$ from water.

The calculated Flory-Huggins parameters for graphene, graphene with hydroxyl group, graphene with carboxyl group, h-BN and $\mathrm{MoS}_{2}$ are 4.81, 3.05, 1.82, 4.45 and 4.26 .
Inkjet printing method. Two types of $2 \mathrm{D}$ material inks were used for inkjet printing: inks of pure-water solvent and inks of $\mathrm{PEO}$ and water mixture. For the PEO-mixed inks, 4 wt. $\%$ of PEO powder (average Mw 1,000,000, Sigma Aldrich) was dissolved in a mixed solvent of $60 \mathrm{wt} . \%$ deionized water and $40 \mathrm{wt} . \%$ ethanol Solutions of PEO in water/ethanol mixture were added to the graphene, $\mathrm{MoS}_{2}$ and h-BN suspension with proper ratio using a THINKY mixing machine (Thinky Inc, ARE-310) to make viscoelastic homogeneous inks.

We used the EHD printing machine (Enjet Inc, Korea) in this experiment. A micro-syringe pump was used to supply the $2 \mathrm{D}$ materials (graphene, $\mathrm{MoS}_{2}$ and h-BN)/PEO composite suspensions from a $1 \mathrm{ml}$ syringe into the metallic nozzle. The nozzle was a $32 \mathrm{G}$ (inner diameter (I.D.): $\sim 0.23 \mathrm{~mm}$, outer diameter (O.D.): $\sim 0.10 \mathrm{~mm}$ ) stainless steel needle. The tip-to-the-collector distance was adjusted at around $2.5 \mathrm{~mm}$ to stabilize the near-field fibre jet to ensure the ohmic flow region. For EHD printing on $\mathrm{Si}$ and $\mathrm{Si} / \mathrm{SiO}_{2}$ substrates, we put them on the metallic movable stage and applied high voltage around $\sim 1.8-2.2 \mathrm{kV}$. Although the $\mathrm{Si} / \mathrm{SiO}_{2}$ substrate has an insulating surface, the inks can be successfully printed by the EHD jet printer, because the insulating layer is thin enough to maintain a high electric field between the nozzle tip and the grounded stage. With the EHD inkjet printing, complicated patterns like letters and meshes could be drawn as shown in Supplementary Fig. 14a-c. To draw a pattern with a pure water inks or on PET substrate, electric field cannot be applied since water is too conductive and PET is insulating. Hence, the ink was pushed merely by the syringe pressure. This method is called nozzle inkjet printing. The printed mesh pattern is shown in Supplementary Fig. 14d.

\section{References}

1. Lee, Y. H. et al. Synthesis of large-area MoS2 atomic layers with chemical vapor deposition. Adv. Mater. 24, 2320-2325 (2012).

2. Li, X. et al. Large-area synthesis of high-quality and uniform graphene films on copper foils. Science 324, 1312-1314 (2009).

3. Song, L. et al. Large scale growth and characterization of atomic hexagonal boron nitride layers. Nano Lett. 10, 3209-3215 (2010).

4. Bae, S. et al. Roll-to-roll production of 30-inch graphene films for transparent electrodes. Nat. Nano 5, 574-578 (2010).

5. Lee, J. H. et al. Wafer-scale growth of single-crystal monolayer graphene on reusable hydrogen-terminated germanium. Science 344, 286-289 (2014).

6. Lee, C., Wei, X., Kysar, J. W. \& Hone, J. Measurement of the elastic properties and intrinsic strength of monolayer graphene. Science 321, 385-388 (2008).

7. Lee, G.-H. et al. Flexible and transparent MoS2 field-effect transistors on hexagonal boron nitride-graphene heterostructures. ACS Nano 7, 7931-7936 (2013).

8. Ciesielski, A. \& Samori, P. Graphene via sonication assisted liquid-phase exfoliation. Chem. Soc. Rev. 43, 381-398 (2014).

9. Paton, K. R. et al. Scalable production of large quantities of defect-free few-layer graphene by shear exfoliation in liquids. Nat. Mater. 13, 624-630 (2014).

10. Hernandez, Y. et al. High-yield production of graphene by liquid-phase exfoliation of graphite. Nat. Nano 3, 563-568 (2008).

11. Lotya, M., King, P. J., Khan, U., De, S. \& Coleman, J. N. High-concentration, surfactant-stabilized graphene dispersions. ACS Nano 4, 3155-3162 (2010).

12. Yi, M., Shen, Z., Zhang, X. \& Ma, S. Achieving concentrated graphene dispersions in water/acetone mixtures by the strategy of tailoring Hansen solubility parameters. J. Phys. D: Appl. Phys. 46, 025301 (2013).

13. Smith, R. J. et al. Large-scale exfoliation of inorganic layered compounds in aqueous surfactant solutions. Adv. Mater. 23, 3944-3948 (2011).

14. Coleman, J. N. Liquid exfoliation of defect-free graphene. Acc. Chem. Res. 46, 14-22 (2013).

15. Coleman, J. N. et al. Two-dimensional nanosheets produced by liquid exfoliation of layered materials. Science 331, 568-571 (2011).

16. Elimelech, M., Jia, X., Gregory, J. \& Williams, R. Particle deposition \& aggregation: measurement, modelling and simulation (Butterworth-Heinemann, 1998).

17. Dikin, D. A. et al. Preparation and characterization of graphene oxide paper. Nature 448, 457-460 (2007).

18. Dreyer, D. R., Park, S., Bielawski, C. W. \& Ruoff, R. S. The chemistry of graphene oxide. Chem. Soc. Rev. 39, 228-240 (2010).

19. Marcano, D. C. et al. Improved synthesis of graphene oxide. ACS Nano 4, 4806-4814 (2010).

20. Zhu, Y. et al. Graphene and graphene oxide: synthesis, properties, and applications. Adv. Mater. 22, 3906-3924 (2010).

21. Silverstein, R. M. Spectrometric identification of organic compounds. J Chem. Educ. 39, 546-557 (1962).

22. Ferrari, A. et al. Raman spectrum of graphene and graphene layers. Phys. Rev. Lett. 97, 187401 (2006).

23. Lee, C. et al. Anomalous lattice vibrations of single-and few-layer MoS2. ACS nano 4, 2695-2700 (2010).

24. Mak, K. F., Lee, C., Hone, J., Shan, J. \& Heinz, T. F. Atomically thin MoS 2: a new direct-gap semiconductor. Phys. Rev. Lett. 105, 136805 (2010).

25. Lin, Y. et al. Aqueous dispersions of few-layered and monolayered hexagonal boron nitride nanosheets from sonication-assisted hydrolysis: critical role of water. J. Phys. Chem. C 115, 2679-2685 (2011). 
26. Bhattacharya, A., Bhattacharya, S. \& Das, G. P. Band gap engineering by functionalization of BN sheet. Phys. Rev. B 85, 035415-1-035415-9 (2012).

27. Suslick, K. S., Hammerton, D. A. \& Cline, R. E. Sonochemical hot spot. J. Am. Chem. Soc. 108, 5641-5642 (1986).

28. Suslick, K. S. Sonochemistry. Science 247, 1439-1445 (1990).

29. Rosenkranz, N., Till, C., Thomsen, C. \& Maultzsch, J. Ab initio calculations of edge-functionalized armchair graphene nanoribbons: structural, electronic and vibrational effects. Phys. Rev. B 84, 195438-1-195438-7 (2011).

30. Pinkerton, J. M. M. The absorption of ultrasonic waves in liquids and its relation to molecular constitution. Proc. Phys. Soc. B 62, 129 (1949).

31. Francois, R. E. \& Garrison, G. R. Sound absorption based on ocean measurements: part I: pure water and magnesium sulfate contributions. J. Acoust. Soc. Am. 72, 896-907 (1982).

32. Antonio Alves Júnior, J. \& Baptista Baldo, J. The behavior of zeta potential of silica suspensions. New J. Glass Ceram. 04, 29-37 (2014).

33. Hunter, R. J. Zeta potential in colloid science: principles and applications (Academic press, 2013).

34. Blasi, P., Giovagnoli, S., Schoubben, A., Ricci, M. \& Rossi, C. Solid lipid nanoparticles for targeted brain drug delivery. Adv. Drug Deliv. Rev. 59, 454-477 (2007).

35. Torrisi, F. \& Coleman, J. N. Electrifying inks with 2D materials. Nat. Nano 9, 738-739 (2014)

36. Zhou, M. et al. Production of graphene by liquid-phase exfoliation of intercalated graphite. Int. J. Electrochem. Sci. 9, 810-820 (2014).

37. Zhang, L. L. et al. Highly conductive and porous activated reduced graphene oxide films for high-power supercapacitors. Nano Lett. 12, 1806-1812 (2012).

38. Hernandez, Y. et al. High-yield production of graphene by liquid-phase exfoliation of graphite. Nat. Nanotech. 3, 563-568 (2008).

39. Lotya, M. et al. Liquid phase production of graphene by exfoliation of graphite in surfactant/water solutions. J. Am. Chem. Soc. 131, 3611-3620 (2009).

40. Reich, S. et al. Resonant Raman scattering in cubic and hexagonal boron nitride. Phys. Rev. B 71, 205201-1-205201-12 (2005).

41. Berkdemir, A. et al. Identification of individual and few layers of WS2 using Raman Spectroscopy. Sci. Rep. 3, 1755 (2013).

42. Tonndorf, P. et al. Photoluminescence emission and Raman response of monolayer MoS2, MoSe2, and WSe2. Opt. Express 21, 4908-4916 (2013).

43. Splendiani, A. et al. Emerging Photoluminescence in Monolayer MoS2. Nano Lett. 10, 1271-1275 (2010).

44. Delley, B. An all-electron numerical method for solving the local density functional for polyatomic molecules. J. Chem. Phys. 92, 508-517 (1990).

45. Delley, B. From molecules to solids with the DMol3 approach. J. Chem. Phys. 113, 7756-7764 (2000).

46. Varshney, V. et al. MD simulations of molybdenum disulphide (MoS 2): force-field parameterization and thermal transport behavior. Comp. Mater. Sci. 48, 101-108 (2010)

47. Morita, Y. et al. Development of a new molecular dynamics method for tribochemical reaction and its application to formation dynamics of MoS2 tribofilm. Appl. Surf. Sci. 254, 7618-7621 (2008).
48. Perdew, J. P., Burke, K. \& Ernzerhof, M. Generalized gradient approximation made simple. Phys. Rev. Lett. 77, 3865-3868 (1996).

49. Delley, B. The conductor-like screening model for polymers and surfaces Mol. Simul. 32, 117-123 (2006).

50. Hansen, C. M. Hansen solubility parameters: a user's handbook (CRC press, 2012).

51. Panayiotou, C. Redefining solubility parameters: the partial solvation parameters. Phys. Chem. Chem. Phys. 14, 3882-3908 (2012).

\section{Acknowledgements}

This study was supported by the Super Computing Centre at UNIST (HPC-UNIST) the Basic Science Research Program (2011-0014209, 2009-0083540, 2011-0015451, 2013R1A1A2007491), the Nano Material Technology Development Program (2012M3A7B4049807), and the Global Frontier Research Centre for Advanced Soft Electronics (2011-0031630, CASE-2014M3A6A5060938) through the National Research Foundation of Korea Grant funded by the Korean government Ministry of Science, ICT and Future Planning.

\section{Author contributions}

J.K. and C.L. developed the idea of 2D material dispersion in water and J.K., S.K., D.-H.C., J.O., S.P. and B.K. carried out the dispersion experiments and contributed to the $\mathrm{pH}$, stability, TEM and spectroscopy measurements. W.-K. and S.W.Y. contributed to the sonic pressure measurements, and H.L. and D.B. carried out inkjet printing. G.H.R. and Z.L. performed HRTEM imaging and its analysis. S.O.P., G.Y.J., E.S. and S.K.K. performed the density functional theory calculations and molecular dynamics simulations. M.C. and T.H.K. contributed to the analysis. J.K., S.K. and C.L. wrote the Letter and all authors provided feedback.

\section{Additional information}

Supplementary Information accompanies this paper at http://www.nature.com/ naturecommunications

Competing financial interests: The authors declare no competing financial interests.

Reprints and permission information is available online at http://npg.nature.com/ reprintsandpermissions/

How to cite this article: Kim, J. et al. Direct exfoliation and dispersion of two-dimensional materials in pure water via temperature control. Nat. Commun. 6:8294 doi: 10.1038/ncomms9294 (2015).

(c) (i) This work is licensed under a Creative Commons Attribution 4.0 International License. The images or other third party material in this article are included in the article's Creative Commons license, unless indicated otherwise in the credit line; if the material is not included under the Creative Commons license, users will need to obtain permission from the license holder to reproduce the material. To view a copy of this license, visit http://creativecommons.org/licenses/by/4.0/ 